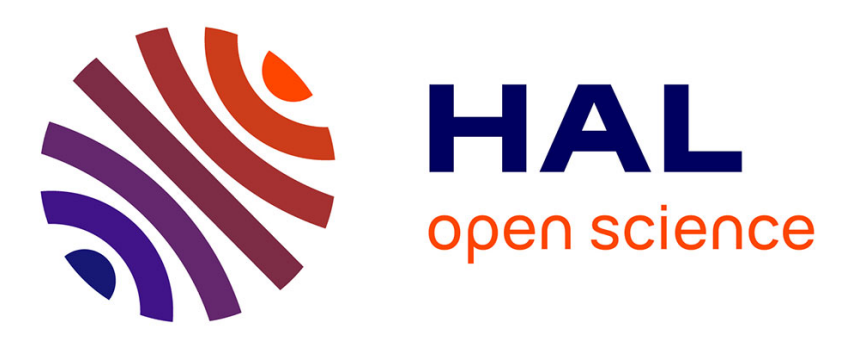

\title{
Torque-Based Balancing for a Humanoid Robot Performing High-Force Interaction Tasks
}

Firas Abi-Farraj, Bernd Henze, Christian Ott, Paolo Robuffo Giordano, Máximo A Roa

\section{- To cite this version:}

Firas Abi-Farraj, Bernd Henze, Christian Ott, Paolo Robuffo Giordano, Máximo A Roa. TorqueBased Balancing for a Humanoid Robot Performing High-Force Interaction Tasks. IEEE Robotics and Automation Letters, 2019, 4 (2), pp.2023-2030. 10.1109/LRA.2019.2898041 . hal-02051454

\section{HAL Id: hal-02051454 \\ https://hal.inria.fr/hal-02051454}

Submitted on 27 Feb 2019

HAL is a multi-disciplinary open access archive for the deposit and dissemination of scientific research documents, whether they are published or not. The documents may come from teaching and research institutions in France or abroad, or from public or private research centers.
L'archive ouverte pluridisciplinaire HAL, est destinée au dépôt et à la diffusion de documents scientifiques de niveau recherche, publiés ou non, émanant des établissements d'enseignement et de recherche français ou étrangers, des laboratoires publics ou privés. 


\title{
Torque-Based Balancing for a Humanoid Robot Performing High-Force Interaction Tasks
}

\author{
Firas Abi-Farraj ${ }^{1}$, Bernd Henze ${ }^{2}$, Christian Ott ${ }^{2}$, Paolo Robuffo Giordano ${ }^{1}$, and Máximo A. Roa ${ }^{2}$
}

\begin{abstract}
Balancing is a critical feature for a robot interacting with an unstructured environment. The balancing control should account for unknown perturbation forces that might destabilize the robot when performing the intended tasks. In the case of humanoid robots this challenge is higher due to the inherent difficulties of balancing a robot on two legs, resulting in a rather small footprint. Approaches for enabling a good balancing behavior on humanoid robots traditionally rely on whole-body balancing approaches. This paper extends a passivity-based whole-body balancing framework to guarantee the equilibrium of a humanoid robot while performing different interaction tasks where the (high) task forces acting on the robot are difficult to foresee. Instead of controlling the center of mass, the proposed controller directly uses information from the Gravito-Inertial Wrench Cone to guarantee the feasibility of the balancing forces. The performance of the approach is validated in a number of successful experimental tests.
\end{abstract}

Index Terms-Humanoid Robots, Force Control

\section{INTRODUCTION}

$\mathbf{H}$ UMANOID robots offer an embodiment that could allow them to enter environments made for humans and utilize standard tools and devices, in order to support and replace humans in dangerous and physically demanding tasks. One example of such expectations on humanoid robots was staged during the DARPA Robotics Challenge (DRC), where robots were required to perform simple tasks in disaster-like scenarios such as driving a car, opening doors, walking on rough terrains, and using simple tools. However, real-life disaster scenarios are even more demanding; one would expect the robot to be capable of moving heavy objects (rocks, debris), operating heavy machinery, and employing tools and devices that require both skill and strength, such as hydraulic rescue tools. Performing such tasks requires suitable control frameworks for dealing with the interaction forces generated during the execution of the task, while still accounting for the balance of the robotic platform.

Accounting for interaction forces of the robot with the environment can be achieved mainly in two different ways.

Manuscript received: September 11, 2018; Revised December 10, 2018; Accepted January 11, 2019.

This paper was recommended for publication by Editor Nikos Tsagarakis upon evaluation of the Associate Editor and Reviewers' comments. This work was supported by the European Union Horizon 2020 Research and Innovation programme under grant agreement No. 645582 (RoMaNS), and H2020-ICT645097 (COMANOID)

${ }^{1}$ The authors are with CNRS, Univ Rennes, Inria, IRISA, Rennes, France. ffiras.abi-farraj,prg\}@irisa.fr

${ }^{2}$ The authors are with the German Aerospace Center (DLR), Institute of Robotics and Mechatronics, 82234 Wessling, Germany. $<$ firstname $>$. <lastname $>$ @dlr.de

Digital Object Identifier (DOI): see top of this page.

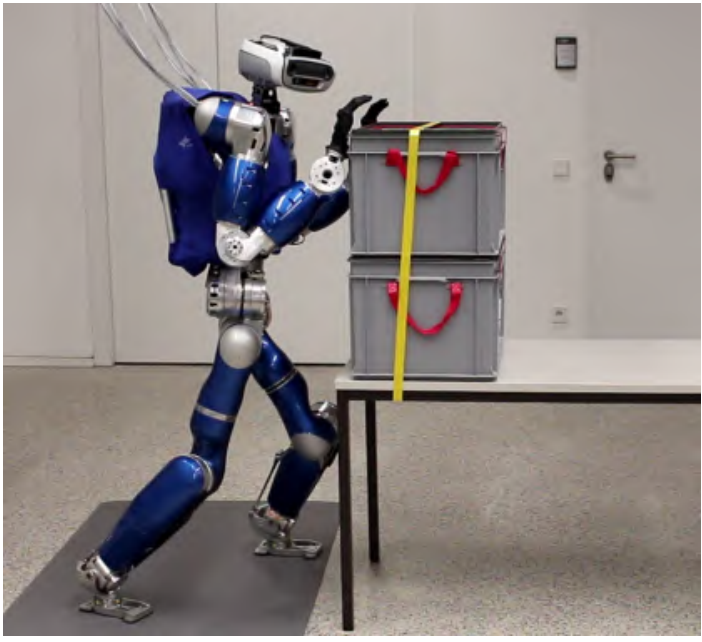

Fig. 1. TORO pushing a table with a weight of $50 \mathrm{~kg}$ on top.

A straightforward approach is to separate interaction and balancing tasks, which usually translates into considering two independent controllers for the lower and upper body of the robot [1], [2]. Forces coming from interactions with the environment using the upper body are considered as a disturbance input by the underlying balancing control, which guarantees the robot balance using only the joints in the lower body. The consideration of force distribution between lower and upper body at the same time can also be achieved through wholebody control frameworks, which exploit the capabilities of redundant robots to deal with multiple tasks [3]-[7]. Different whole-body balancing controllers have been proposed based mainly on two approaches: solving the inverse kinematics or dynamics of the robot [3], [8]-[10], or using passivity-based approaches [5], [11], [12]. A subset of the whole-body control frameworks feature a hierarchical architecture that allows for multiple control objectives [3], [6], [13]. In [7], a hierarchical approach was used to balance on contacts scattered over the whole body of the robot.

When it is foreseen that high forces could arise in the interaction of the robot with the environment, the robot can plan in advance the best posture and force distribution required to deal with the task [14]. The planning has to consider several factors such as kinematic limitations, actuator constraints, or characteristics of the supporting contacts. A recent method to account for the contact properties is based on the computation of the so-called Gravito-Inertial Wrench Cone (GIWC), which provides the maximum perturbations that the robot can resist at a given configuration, and/or the maximum interaction force that the robot can generate at a given posture [15]-[18] 
However, this polyhedron has been so far used for (offline) planning and not for instantaneous control of the robot posture. In this context, the main goal of this paper is to leverage the concept of the GIWC and apply it to instantaneous control of the robot posture. A whole-body controller usually requires an explicit goal provided for the center of mass (CoM) trajectory. This paper does not specify an explicit location for the CoM; instead, the CoM is moved such that the contact wrenches required for balancing the interaction force are feasible for each contact configuration. In that way, the polyhedron of feasible balancing wrenches or GIWC is used to ensure the online stability of the humanoid (with respect to the contact properties) while interacting with an unknown environment.

\section{PRELIMINARIES AND BACKGROUND}

\section{A. Dynamic Model}

In the field of legged humanoid robotics it is common to use dynamic models with a free-floating base, as they provide a higher flexibility regarding contact changes compared to dynamic models with a fixed base. In general, a central body within the kinematic structure of the robot is chosen as base link, e.g. the hip or the trunk. Some works also employ the center of mass (CoM) as base, since it represents an essential point for balancing. Here, we will follow [5] by defining a CoM frame $\mathcal{C}$, which is located at the CoM and has the same orientation of the hip. Let $\boldsymbol{x}_{c} \in \mathbb{R}^{3}$ and $\boldsymbol{R}_{c} \in \mathcal{S O}(3)$ denote the position and orientation of the frame $\mathcal{C}$ with respect to the world frame $\mathcal{W}$. The corresponding translational and rotational velocities are given by $\dot{\boldsymbol{x}}_{c}$ and $\boldsymbol{\omega}_{c}$, respectively. Considering the $n$ joint angles $\boldsymbol{q} \in \mathbb{R}^{n}$ and $\boldsymbol{v}_{c}=\left(\dot{\boldsymbol{x}}_{c}^{T}, \boldsymbol{\omega}_{c}^{T}\right)^{T}$, the dynamics of the humanoid robot is given by

$$
\boldsymbol{M} \underbrace{\left(\begin{array}{c}
\dot{\boldsymbol{v}}_{c} \\
\ddot{\boldsymbol{q}}
\end{array}\right)}_{\dot{\boldsymbol{\nu}}}+\boldsymbol{C} \underbrace{\left(\begin{array}{c}
\boldsymbol{v}_{c} \\
\dot{\boldsymbol{q}}
\end{array}\right)}_{\boldsymbol{\nu}}+\underbrace{\left(\begin{array}{c}
m \boldsymbol{g}_{0} \\
\mathbf{0}
\end{array}\right)}_{\boldsymbol{g}}=\left(\begin{array}{c}
\mathbf{0} \\
\boldsymbol{\tau}
\end{array}\right)+\boldsymbol{\tau}_{\mathrm{ext}} .
$$

Herein, $\boldsymbol{M} \in \mathbb{R}^{(6+n) \times(6+n)}$ and $\boldsymbol{C} \in \mathbb{R}^{(6+n) \times(6+n)}$ represent the inertia and Coriolis/centrifugal matrix, respectively. The gravitational torques are given by $\boldsymbol{g} \in \mathbb{R}^{6+n}$, with $m$ denoting the total mass of the robot and $\boldsymbol{g}_{0} \in \mathbb{R}^{6}$ the gravitational acceleration $^{1}$. The joint torques are denoted by $\tau \in \mathbb{R}^{n}$. The influence of external wrenches acting on the robot is taken into account by the generalized torque vector $\tau_{\text {ext }} \in \mathbb{R}^{6+n}$.

Let us divide the $\Psi$ end-effectors into two subgroups as in [5]: the first group is referred to as "balancing end-effectors" (bal) and contains the $\psi$ end-effectors that are used by the robot to support itself (usually the feet). The remaining endeffectors are called "interaction end-effectors" (int), because they can still be used for a manipulation or interaction task (usually the hands). With this distinction, the Cartesian velocities of the end-effectors $\boldsymbol{v} \in \mathbb{R}^{6 \Psi}$ are given by

$$
\boldsymbol{v}=\left(\begin{array}{l}
\boldsymbol{v}_{\text {bal }} \\
\boldsymbol{v}_{\text {int }}
\end{array}\right)=\left[\begin{array}{l}
\boldsymbol{J}_{\text {bal }} \\
\boldsymbol{J}_{\text {int }}
\end{array}\right] \boldsymbol{\nu}=\boldsymbol{J} \boldsymbol{\nu}
$$

with the Jacobian matrix $\boldsymbol{J} \in \mathbb{R}^{6 \Psi \times(6+n)}$ and $\boldsymbol{v}_{\text {bal }} \in \mathbb{R}^{6 \psi}$, $\boldsymbol{v}_{\text {int }} \in \mathbb{R}^{6(\Psi-\psi)}, \boldsymbol{J}_{\text {bal }} \in \mathbb{R}^{6 \psi \times(6+n)}, \boldsymbol{J}_{\text {int }} \in \mathbb{R}^{6(\Psi-\psi) \times(6+n)}$. If

\footnotetext{
${ }^{1}$ Note that $g_{0}$ is a six-dimensional vector, containing three translational and three rotational DoFs of the CoM frame $\mathcal{C}$.
}

all external disturbances act solely at the end-effectors, $\boldsymbol{\tau}_{\text {ext }}$ simplifies to

$$
\boldsymbol{\tau}_{\mathrm{ext}}=\boldsymbol{J}^{T} \boldsymbol{F}_{\mathrm{ext}}
$$

\section{B. Overview of the Compliant Balancing Controller}

This section provides a brief summary of our balancing controller, presented in detail in [5]. The controller stabilizes the CoM by a Cartesian compliance, which generates a wrench $\boldsymbol{F}_{c} \in \mathbb{R}^{6}$ at the CoM frame $\mathcal{C}$. Each one of the interaction end-effectors is stabilized by another Cartesian compliance, with the resulting wrenches stacked into $\boldsymbol{F}_{\text {int }} \in$ $\mathbb{R}^{6(\Psi-\psi)}$. In order to support the robot, the control algorithm computes a suitable set of balancing wrenches $\boldsymbol{F}_{\text {bal }}^{\mathrm{opt}} \in \mathbb{R}^{6 \Psi}$ by minimizing the following quadratic optimization problem

$$
\boldsymbol{F}_{\text {bal }}^{\text {opt }}=\underset{\boldsymbol{F}_{\text {bal }}}{\operatorname{argmin}}\left(\boldsymbol{F}_{\text {bal }}-\boldsymbol{F}_{\text {bal }}^{\text {def }}\right)^{T} \boldsymbol{Q}\left(\boldsymbol{F}_{\text {bal }}-\boldsymbol{F}_{\text {bal }}^{\text {def }}\right)
$$

with respect to

$$
\boldsymbol{A} \boldsymbol{d}_{\mathrm{bal}}^{T} \boldsymbol{F}_{\mathrm{bal}}+\boldsymbol{A} \boldsymbol{d}_{\mathrm{int}}^{T} \boldsymbol{F}_{\text {int }}=m \boldsymbol{g}_{0}-\boldsymbol{F}_{c}
$$

and

$$
\begin{aligned}
f_{k, z} & \geq f_{k, z}^{\min }, \\
\delta_{k, x / y}^{\min } & \leq \delta_{k, x / y} \leq \delta_{k, x / y}^{\max }, \\
\left|f_{k, x / y}\right| & \leq \tilde{\mu}_{k} f_{k, z} .
\end{aligned}
$$

The cost function (4) minimizes the deviation of $\boldsymbol{F}_{\text {bal }}$ from a default wrench distribution ${ }^{2} \boldsymbol{F}_{\text {bal }}^{\text {def }}$ based on the positive definite weighting matrix $Q \in \mathbb{R}^{6 \psi \times 6 \psi}$. The equality constraint (5) represents the underactuation of the base by demanding that the influence of all commanded end-effector wrenches $\left(\boldsymbol{F}_{\text {bal }}\right.$, $\boldsymbol{F}_{\text {int }}$ ) on the CoM must sum up to the compliance wrench $\boldsymbol{F}_{c}$ plus gravity. For this, the Jacobian matrices $\boldsymbol{J}_{\text {bal }}=\left[\boldsymbol{A} \boldsymbol{d}_{\text {bal }} \overline{\boldsymbol{J}}_{\text {bal }}\right]$ and $\boldsymbol{J}_{\text {int }}=\left[\boldsymbol{A d}_{\text {int }} \overline{\boldsymbol{J}}_{\text {int }}\right]$ are divided into $\boldsymbol{A d}_{\text {bal }} \in \mathbb{R}^{6 \psi \times 6}$, $\boldsymbol{A d}_{\text {int }} \in \mathbb{R}^{6(\Psi-\psi) \times 6}, \overline{\boldsymbol{J}}_{\text {bal }} \in \mathbb{R}^{6 \psi \times n}$, and $\overline{\boldsymbol{J}}_{\text {int }} \in \mathbb{R}^{6(\Psi-\psi) \times n}$. The first two are the stacked adjoint matrices of each endeffector, which relate a motion of the CoM frame $\mathcal{C}$ with a motion of the end-effectors. The matrices $\overline{\boldsymbol{J}}_{\text {bal }}$ and $\overline{\boldsymbol{J}}_{\text {int }}$ map a motion of the joints to a motion of the end-effectors. The inequality constraints (6) describe the contact model to which $\boldsymbol{F}_{\text {bal }}$ is subjected to in order to account for unilaterality, location of the Center of Pressure (CoP), and friction of the balancing contacts. For each end-effector wrench within $\boldsymbol{F}_{\text {bal }}$, the force perpendicular to the contact surface has a lower bound given as a minimum contact force $f_{k, z}^{\min }$ in order to prevent the end-effector from lifting off ${ }^{3}$. Slippage is prevented by constraining the tangential forces $f_{k, x / y}$ to the friction cone given by $\tilde{\mu}_{k}$. The CoP $\delta_{k}$ is constrained to the interior of the contact surface via $\delta_{k, x / y}^{\min / \max }$ to prevent the end-effector from tilting.

After computing a suitable wrench distribution $\boldsymbol{F}_{\text {bal }}^{\text {opt }}$, the end-effector wrenches are mapped to joint space via

$$
\boldsymbol{\tau}=-\left[\begin{array}{ll}
\overline{\boldsymbol{J}}_{\text {bal }}^{T} & \overline{\boldsymbol{J}}_{\text {int }}^{T}
\end{array}\right]\left(\begin{array}{c}
\boldsymbol{F}_{\text {bal }}^{\text {opt }} \\
\boldsymbol{F}_{\text {int }}
\end{array}\right) .
$$

\footnotetext{
${ }^{2}$ The default distribution $\boldsymbol{F}_{\mathrm{bal}}^{\mathrm{def}}$ is a tunable parameter, which can be set for instance to $50 \%$ of the weight of the robot per foot if the posture is symmetric.

${ }^{3}$ For the conducted experiments (section IV), $f_{k, z}^{\min }$ was set to $50 \mathrm{~N}$ in order to account for the effect of joint friction.
} 


\section{Controller Design}

In the architecture described in [5], a Cartesian compliance defines the wrench $\boldsymbol{F}_{c}$ to be applied at the CoM in order to stabilize it at a desired configuration. This approach proved efficient for balancing, and showed significant robustness to noise and external disturbances. However, the amount of force and torque the robot can apply with the interaction endeffectors is limited because the balancing contacts must provide an appropriate counter-force constrained by the contact model (6). With that in mind, a new approach for controlling the wrench $\boldsymbol{F}_{c}$ on the CoM while accounting for the interaction forces between the robot and the environment is proposed in this paper. The proposed architecture is based on the controller presented in [5] and aims at ensuring the feasibility of the needed balancing wrenches as a function of the contact configuration of the balancing end-effectors by moving the CoM accordingly. The control is based on the polyhedron of feasible balancing wrenches calculated from the current contact configuration, with no need for calculating the support polygon. The actuator limitations are not considered in the described controller, but their inclusion is an interesting direction for future exploration.

\section{A. Force Polyhedron and Support Polygon}

The support polygon of a humanoid robot is the region in which the vertical projection of the CoM must lie so that the balancing end-effectors can carry statically the robot's weight. In case that the humanoid is moving on a horizontal plane, the support polygon $\mathcal{S}$ is the convex hull of the contact areas with the ground, mainly corresponding to the feet of the humanoid (Fig. 2). However, in a multi-contact scenario where other parts of the humanoid's body are used for balancing (e.g. the hands), the calculation of the support polygon gets more complicated [19]. The same holds in the case of non-horizontal or non-planar contact surfaces (e.g. rough terrain).

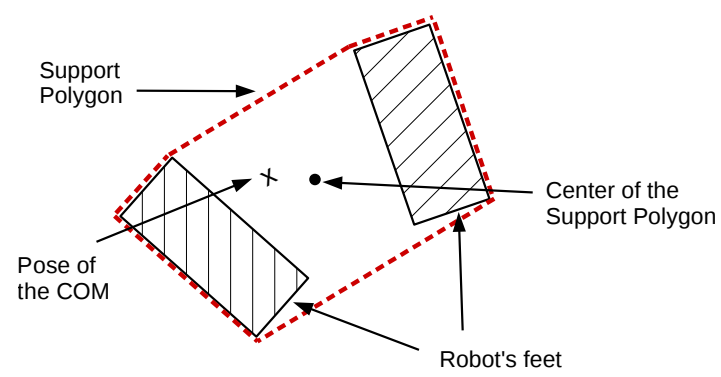

Fig. 2. Illustration of the support polygon for a robot standing on horizontal ground.

The support polygon can be calculated from the GIWC, which is the set of wrenches that the balancing end-effectors can apply at a particular contact configuration. The contact configuration, referred to hereafter by $\Upsilon$, is the pose of the end-effectors used for balancing in the world frame. It is dependent on the contact configuration itself and the contact model (see eq. (6)) specifying the maximum load that each end-effector can carry. The contact model can be re-written into a polyhedron $\boldsymbol{\xi}_{i}=\left\{\boldsymbol{F}_{\text {bal, } \mathrm{i}}: \boldsymbol{A}_{i} \boldsymbol{F}_{\text {bal,i }} \leq \boldsymbol{b}_{i}\right\}$ representing the set of feasible wrenches for each balancing end-effector, where $\boldsymbol{F}_{\text {bal,i }} \in \mathbb{R}^{6}$ (see [16] for details). The GIWC can be calculated via the Minkowski-Sum of $\boldsymbol{\xi}_{i}$, resulting in the wrench polyhedron $\boldsymbol{\xi}$ defined as

$$
\boldsymbol{\xi}=\left\{{ }^{w} \boldsymbol{F}_{\text {bal, }}: \boldsymbol{A}^{w} \boldsymbol{F}_{\text {bal }, \mathrm{t}} \leq \boldsymbol{b}\right\}
$$

where $\boldsymbol{A}=\left[\begin{array}{lll}\boldsymbol{A}_{1} & \ldots & \boldsymbol{A}_{n}\end{array}\right]^{T} \in \mathbb{R}^{m \times 6}$ is a constant matrix and $\boldsymbol{b}=\left(\begin{array}{lll}b_{1} & \ldots & b_{m}\end{array}\right)^{T} \in \mathbb{R}^{m}$ a constant vector. The total wrench ${ }^{w} \boldsymbol{F}_{\text {bal, } t} \in \mathbb{R}^{6}$ is defined in the world frame $\mathcal{W}$ and is related to $\boldsymbol{F}_{\text {bal,i }}$ via ${ }^{w} \boldsymbol{F}_{\text {bal, }}=\boldsymbol{A d}_{c}^{T} \boldsymbol{A} \boldsymbol{d}_{\text {bal }}^{T} \boldsymbol{F}_{\text {bal }}$. The adjoint matrix $\boldsymbol{A} \boldsymbol{d}_{c}$ maps the total wrench from the CoM frame to the world frame. Note that the resulting matrix ${ }^{w} \boldsymbol{A} \boldsymbol{d}_{\text {bal }}=\boldsymbol{A} \boldsymbol{d}_{\text {bal }} \boldsymbol{A} \boldsymbol{d}_{c}$ is constant, as the balancing contacts are stationary with respect to the world frame $\mathcal{W}$. Any wrench ${ }^{w} \boldsymbol{F}$ satisfying (8) is feasible and can be applied by the balancing end-effectors in the current contact configuration $\boldsymbol{\Upsilon}$. If $\boldsymbol{\Upsilon}$ changes, $\boldsymbol{\xi}$ can be re-computed accordingly. We refer the reader to [16] for more details on the calculation of the GIWC.

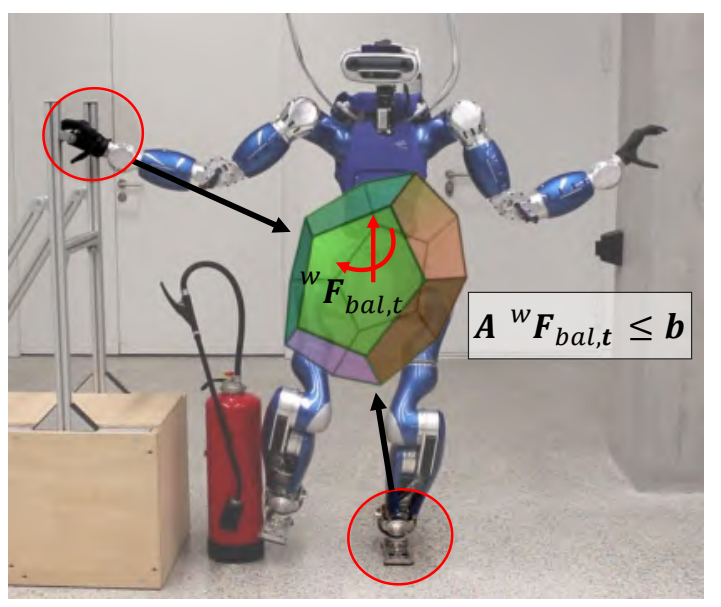

Fig. 3. TORO balancing with its right hand and left foot while interacting with the environment using its right foot. The two balancing end-effectors can exert a set of forces estimated by the polyhedron $\boldsymbol{A}^{w} \boldsymbol{F}_{\text {bal, }} \leq \boldsymbol{b}$.

The support polygon is calculated at a particular pre-defined point by projecting $\boldsymbol{\xi}$ on a certain plane of interest [17]. For instance, in the case of a robot standing on horizontal ground, $\boldsymbol{\xi}$ is projected on the horizontal plane at a point located on the ground to retrieve the support polygon. This solution is, however, clearly suboptimal since $\boldsymbol{\xi}$ corresponds to a whole polyhedron (8), and is not just restricted to a planar projection. Therefore in this paper we propose to define a control law at the level of the balancing wrench itself, without the need of calculating the support polygon.

\section{B. Proposed Controller}

Assuming a quasi-static scenario $\left(F_{c} \approx 0\right)$ and that all external forces/torques act only at the end-effectors, eq. (5) describing the forces at the $\mathrm{CoM}$ and defined at the origin of the world frame $\mathcal{W}$ can then be written as

$$
{ }^{w} \boldsymbol{F}_{\text {bal }, \mathrm{t}}+{ }^{w} \boldsymbol{A} \boldsymbol{d}_{\mathrm{int}}^{T} \boldsymbol{F}_{\mathrm{int}}+{ }^{w} \boldsymbol{A} \boldsymbol{d}_{c}^{T} m \boldsymbol{g}_{0}=0 .
$$




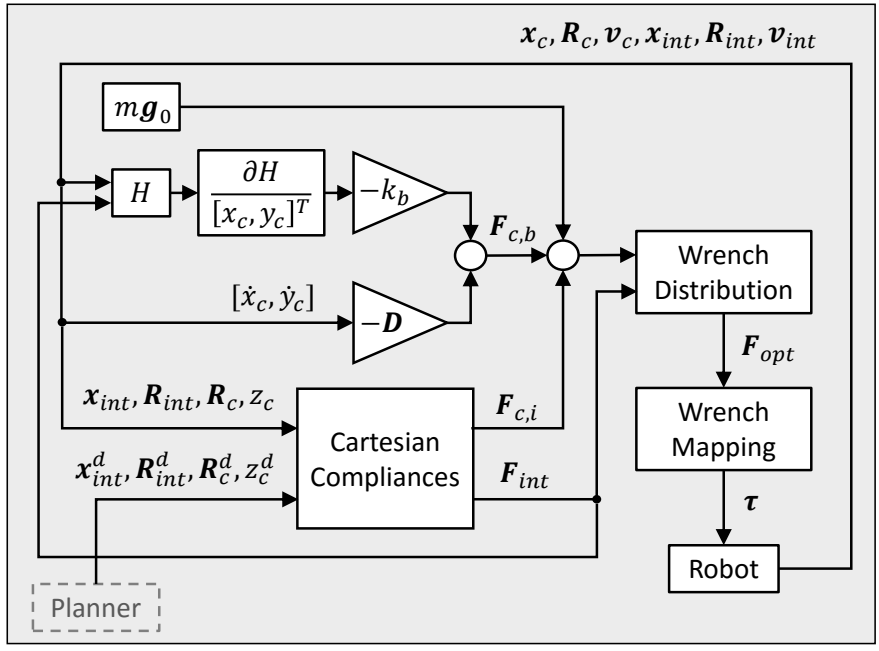

Fig. 4. Overview of the control architecture.

Note that $\boldsymbol{F}_{\text {int }}$ represents the commanded wrenches of the compliances arising from the interaction task. The adjoint matrices ${ }^{w} \boldsymbol{A} \boldsymbol{d}_{c}$ and ${ }^{w} \boldsymbol{A} \boldsymbol{d}_{\text {int }}$ are a function of the CoM location $\boldsymbol{x}_{c}$ and of the position $\boldsymbol{x}_{\mathrm{int,i}}$ of each interaction end-effector, respectively. From the definition of $\boldsymbol{\xi}$ in (8), the balancing wrenches are feasible if

$$
\boldsymbol{A}^{w} \boldsymbol{F}_{\text {bal, }} \leq \boldsymbol{b}
$$

as detailed in [16].

Assuming that the robot starts from a feasible configuration, we define a "distance" measure $d_{i}$ from the current balancing wrench ${ }^{w} \boldsymbol{F}_{\text {bal, t }}$ to the $i_{t h}$ face of the polyhedron $\boldsymbol{\xi}$ given by $\left\{\boldsymbol{F}_{\text {bal,i }}: \boldsymbol{A}_{i} \boldsymbol{F}_{\text {bal,i }}=\boldsymbol{b}_{i}\right\}$, such that

$$
d_{i}\left({ }^{w} \boldsymbol{F}_{\text {bal }, \mathrm{t}}\right)=\frac{\boldsymbol{b}_{i}-\boldsymbol{A}_{i}{ }^{w} \boldsymbol{F}_{\text {bal, } \mathrm{t}} .}{\left\|\boldsymbol{A}_{i}\right\|} .
$$

While there are infinite ways of expressing the GIWC, the measure $d_{i}$ is unique and scale invariant. We however assume that there are no duplicate surfaces in (10).

On the other hand, from (9) we know that

$$
\begin{aligned}
{ }^{w} \boldsymbol{F}_{\text {bal }, \mathrm{t}} & =-{ }^{w} \boldsymbol{A} \boldsymbol{d}_{\text {int }}^{T} \boldsymbol{F}_{\text {int }}-{ }^{w} \boldsymbol{A} \boldsymbol{d}_{c}^{T} m \boldsymbol{g}_{0} \\
& =-{ }^{w} \boldsymbol{A} \boldsymbol{d}_{\text {int }}^{T} \boldsymbol{F}_{\text {int }}-\left[\begin{array}{cc}
\boldsymbol{I}_{3 \times 3} & \mathbf{0}_{3 \times 3} \\
{\left[\boldsymbol{x}_{c}\right]_{x}} & \boldsymbol{I}_{3 \times 3}
\end{array}\right] m \boldsymbol{g}_{0} \\
& =-{ }^{w} \boldsymbol{A} \boldsymbol{d}_{\text {int }}^{T} \boldsymbol{F}_{\text {int }}-\left[\begin{array}{c}
m \boldsymbol{g}_{0, x} \\
\mathbf{0}_{3 \times 3}
\end{array}\right]+\left[\begin{array}{c}
\mathbf{0}_{3 \times 3} \\
{\left[m \boldsymbol{g}_{0, x}\right]_{x}}
\end{array}\right] \boldsymbol{x}_{c} \\
& =-{ }^{w} \boldsymbol{A} \boldsymbol{d}_{\text {int }}^{T} \boldsymbol{F}_{\text {int }}-\left[\begin{array}{c}
m \boldsymbol{g}_{0, x} \\
\mathbf{0}_{3 \times 3}
\end{array}\right]+m g\left[\begin{array}{cc}
\mathbf{0}_{3 \times 2} \\
{\left[\begin{array}{cc}
0 \\
1 & 0
\end{array}\right]} \\
\mathbf{0}_{1 \times 3}
\end{array}\right]\left[\begin{array}{l}
x_{c} \\
y_{c}
\end{array}\right],
\end{aligned}
$$

where $[\cdot]_{x}$ denotes the skew-symmetric operator, and $\boldsymbol{g}_{0, x} \in \mathbb{R}^{3}$ is the linear component of $\boldsymbol{g}_{0}=\left(\boldsymbol{g}_{0, x}^{T} \mathbf{0}_{3}\right)^{T}$, with $\boldsymbol{g}_{0, x}^{T}=\left[\begin{array}{lll}0 & 0 & -g\end{array}\right]^{T}$. As no previous knowledge of the environment is assumed, $\boldsymbol{F}_{\text {int }}$ in the above equation is unknown, which leaves $x_{c}$ and $y_{c}$ as the only controllable variables.

The balancing end-effectors can apply the required balancing wrench ${ }^{w} \boldsymbol{F}_{\text {bal,t }}$ if and only if $d_{i}>0 \forall i$. We then define a potential $H$ to encode the proximity of the balancing wrench to the limits of $\boldsymbol{\xi} . H$ needs to be a monotone function that grows infinite as $d \rightarrow 0$, in order to reflect the loss of balance in such a case. Among the many ways to design such a potential, we empirically choose $H$ to be

$$
H\left(x_{c}, y_{c}\right)=\sum_{i} \frac{1}{d_{i}\left(x_{c}, y_{c}\right)} .
$$

To simplify the notation, $H\left(x_{c}, y_{c}\right)$ and $d_{i}\left(x_{c}, y_{c}\right)$ are respectively referred to by $H$ and $d_{i}$ in the rest of the paper.

Since $\boldsymbol{\xi}$ is convex, the balance of the humanoid robot can be ensured by defining a gradient-descent control law on $H$ (Fig. 4). The gradient of $H$ with respect to $\left[x_{c}, y_{c}\right]^{T}$ can be written as

$$
\frac{\partial H}{\partial\left[x_{c}, y_{c}\right]^{T}}=m g\left(\sum_{i} \frac{\boldsymbol{A}_{i}}{d_{i}^{2}\left\|\boldsymbol{A}_{i}\right\|}\right)\left[\begin{array}{c}
\mathbf{0}_{3 \times 2} \\
{\left[\begin{array}{cc}
0 & -1 \\
1 & 0 \\
\mathbf{0}_{1 \times 3}
\end{array}\right]}
\end{array}\right]
$$

As the only CoM parameters impacting $H$ are $x_{c}$ and $y_{c}$, the control wrench $\boldsymbol{F}_{c}$ to be applied at the CoM (eq. (5)) can be divided into two parts $\boldsymbol{F}_{c}=\left[\begin{array}{ll}\boldsymbol{F}_{c, b} & \boldsymbol{F}_{c, i}\end{array}\right]^{T}$, where $\boldsymbol{F}_{c, b}=\left[\begin{array}{ll}F_{c, x} & F_{c, y}\end{array}\right]^{T} \in \mathbb{R}^{2}$ ensures the balance of the robot by acting on the $x$ and $y$ components of $\boldsymbol{x}_{c}$, while $\boldsymbol{F}_{c, i}=\left[F_{c, z} \boldsymbol{F}_{c, \boldsymbol{\omega}}\right]^{T} \in \mathbb{R}^{4}$ commands the $z$ component of $\boldsymbol{x}_{c}$ and the orientation $\boldsymbol{R}_{c}$ of the CoM frame $\mathcal{C}$. Consequently, we define $\boldsymbol{F}_{c, b}$ as

$$
\boldsymbol{F}_{c, b}=-k_{b}\left(\frac{\partial H}{\partial\left[x_{c} y_{c}\right]^{T}}\right)^{T}-\boldsymbol{D}_{c, b}\left[\begin{array}{c}
\dot{x}_{c} \\
\dot{y}_{c}
\end{array}\right],
$$

where $\boldsymbol{D}_{c, b} \in \mathbb{R}^{2 \times 2}$ is a damping matrix, and $k_{b}$ is a control gain. Note that while the interaction forces, $\boldsymbol{F}_{\text {int }}$, cannot be predicted, they are accounted for in the term $1 / d_{i}^{2}$ in $\partial H / \partial\left[\begin{array}{ll}x_{c} & y_{c}\end{array}\right]^{T}$. It is also important to indicate that $\boldsymbol{F}_{\text {int }}$ actually corresponds to the commanded forces on the interacting end-effectors (which are controlled in compliance), and it is not retrieved from the measured torques. On the other hand, $\boldsymbol{F}_{c, i}$ is defined as an impedance-based control task to allow the control of $z_{c}$ and $\boldsymbol{R}_{c}$ as described in section II-B.

\section{Contact Switching}

While a humanoid can balance on its feet, different contact configurations may be required in cases where, for example, the ground is not planar or where the robot needs to use its feet to interact with the environment. Switching from a particular contact configuration to another one requires shifting the supporting forces from some end-effectors to others, depending on the given contact configuration. In this section, we describe an autonomous contact-switching algorithm that handles this "weight shifting" process in the wrench space when the endeffectors are already in position. A typical situation happens when the two feet of the robot are on the ground and one hand is in contact with a wall. The robot can switch contacts to balance using any possible combination of the three endeffectors, i.e. the two feet, the two feet and the hand, or one foot and the hand. The three end-effectors are assumed to be static during the switching process. In order to handle cases 
where the balancing end-effectors should be moved (e.g. for stepping), this algorithm needs to be topped with a suitable planner.

Assume the robot is balancing on a particular contact configuration $\boldsymbol{\Upsilon}_{1}$, with $\psi_{1}$ end-effectors, and needs to switch to a different contact configuration $\boldsymbol{\Upsilon}_{2}$, with $\psi_{2}$ end-effectors. Two GIWC, $\boldsymbol{\xi}_{1}$ and $\boldsymbol{\xi}_{2}$, can be defined corresponding to contact configurations $\boldsymbol{\Upsilon}_{1}$ and $\boldsymbol{\Upsilon}_{2}$ respectively. The goal of the contact switch is to 'move' ${ }^{w} \boldsymbol{F}_{\mathrm{bal}, t}$ from polyhedron $\boldsymbol{\xi}_{1}$ to polyhedron $\xi_{2}$. To this end, we retrieve the optimal CoM position, $\left[x_{c}, y_{c}\right]_{o p t, x}$, corresponding to each polyhedron $\boldsymbol{\xi}_{x}$ by solving

$$
\min _{\left[x_{c}, y_{c}\right]} H_{\boldsymbol{\xi}_{x}}
$$

where $H_{\boldsymbol{\xi}_{x}}$ is the potential in (13) calculated for the GIWC $\boldsymbol{\xi}_{x} .\left[x_{c}, y_{c}\right]_{o p t, x}$ can then be substituted in (12) to retrieve the optimal balancing wrench ${ }^{w} \boldsymbol{F}_{\text {opt }, x}$ corresponding to $\boldsymbol{\xi}_{x}$. Note that when the robot is in contact configuration $\boldsymbol{\xi}_{x}$, the gradient descent controller defined in section II-B maintains ${ }^{w} \boldsymbol{F}_{\text {bal }, t}$ at ${ }^{w} \boldsymbol{F}_{\text {opt }, x}$, which ensures a smooth back and forth switch between the two controllers.

The contact-switching controller aims then to drive ${ }^{w} \boldsymbol{F}_{\text {bal }, t}$ from ${ }^{w} \boldsymbol{F}_{\mathrm{opt}, 1}$ to ${ }^{w} \boldsymbol{F}_{\mathrm{opt}, 2}$ (i.e. from polyhedron $\boldsymbol{\xi}_{1}$ to polyhedron $\left.\xi_{2}\right)$. To this end, we define a quadratic contact-switch potential $H_{c s}$ as

$$
H_{c s}=\left({ }^{w} \boldsymbol{F}_{\text {bal }, d}(t)-{ }^{w} \boldsymbol{F}_{\text {bal }}\right)^{T}\left({ }^{w} \boldsymbol{F}_{\text {bal }, d}(t)-{ }^{w} \boldsymbol{F}_{\text {bal }}\right)
$$

where ${ }^{w} \boldsymbol{F}_{\text {bal }, d}=(1-\alpha){ }^{w} \boldsymbol{F}_{\mathrm{opt}, 1}+\alpha{ }^{w} \boldsymbol{F}_{\mathrm{opt}, 2}$ is the desired balancing wrench that shifts smoothly from ${ }^{w} \boldsymbol{F}_{\text {opt }, 1}$ to ${ }^{w} \boldsymbol{F}_{\mathrm{opt}, 2}$ as $\alpha: 0 \rightarrow 1$. Since the contacts corresponding to both $\boldsymbol{\xi}_{1}$ and $\boldsymbol{\xi}_{2}$ are established during the switch, and as all the computed wrench polyhedrons are convex, ${ }^{w} \boldsymbol{F}_{\text {bal }, d}$ necessarily lies within the Minkowski-Sum of $\boldsymbol{\xi}_{1}$ and $\boldsymbol{\xi}_{2}$, which ensures its feasibility.

Finally, and similarly to section II-B, the wrench $\boldsymbol{F}_{c, c s}$ to be applied at the CoM is defined as

$$
\boldsymbol{F}_{c, c s}=-k_{c s}\left(\frac{\partial H_{c s}}{\partial\left[x_{c} y_{c}\right]^{T}}\right)^{T}-\boldsymbol{D}_{c, b}\left[\begin{array}{c}
\dot{x}_{c} \\
\dot{y}_{c}
\end{array}\right]
$$

where $k_{c s}$ is a control gain. The resulting overall algorithm is detailed in Alg. 1. For this algorithm, $\epsilon_{\alpha}$ and $\epsilon_{H_{c s}}$ are control variables to be tuned.

\section{EXPERIMENTS AND RESULTS}

\section{A. Experimental Setup}

The proposed approach was implemented on TORO, the torque-controlled humanoid robot developed at DLR [20]. The robot has $27 \mathrm{DoF}$ (plus $12 \mathrm{DoF}$ at the hands), a total height of $1.74 \mathrm{~m}$ and a weight of $76.4 \mathrm{~kg}$. 25 of the joints, located at the arms, legs and hip, are based on the DLR-KUKA LBR (Lightweight robot arm), and allow for both position and torque control modes. Besides position and torque sensing at the LBR-based joints, TORO has an IMU at the trunk and a 6-DoF Force-Torque sensor at each foot.

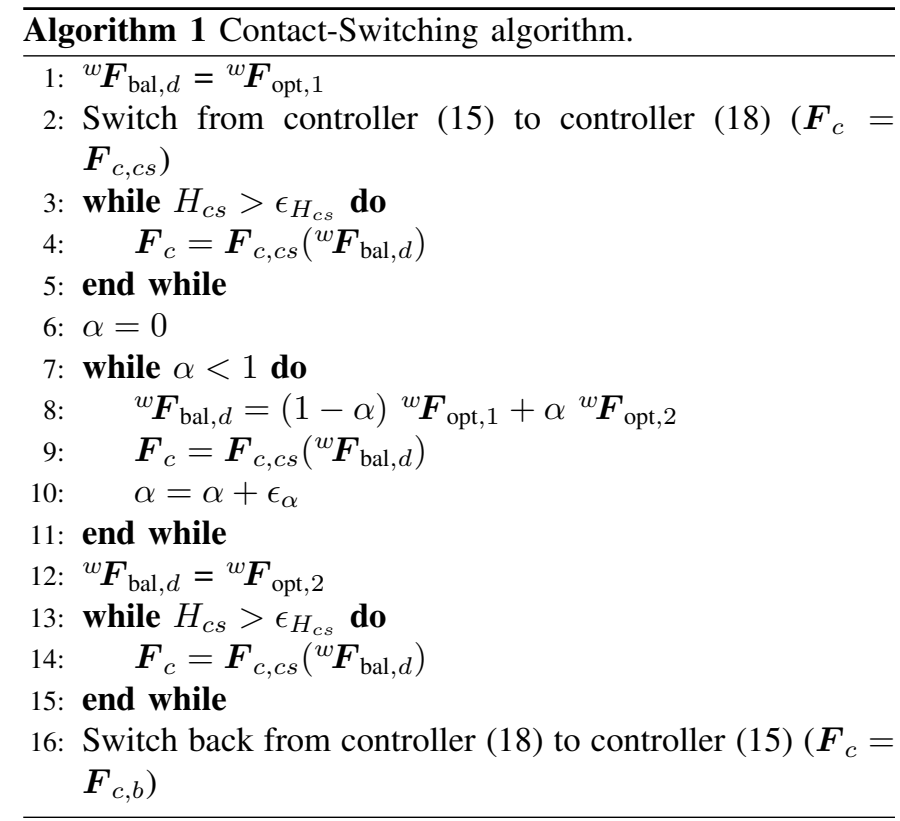

\section{B. Experimental results}

Several experiments were performed to validate the proposed approach. They are demonstrated in the attached video (available also at https://youtu.be/TB149-XvnRE). In this section, we discuss four experiments that demonstrate the applicability of the proposed architecture for balancing while interacting with the environment, and for automatic contact switching.

1) Experiment 1. Carrying a heavy box: The experiment consists of a person passing to the robot a box weighing $25 \mathrm{~kg}$ (nearly one third of the robot's weight), and then taking it back. The hands of the robot are controlled in impedance mode and are subject to the Cartesian end-effector compliance described in section II-B. No feed-forward forces were fed to the arms to counteract the weight of the box, which is totally unknown to the controller. Figure 5 shows the results of the experiment. The two dashed lines denote the instances when the robot takes the box from the human $(\mathrm{t}=6.5 \mathrm{~s})$ and when it gives it back $(\mathrm{t}=19.5 \mathrm{~s})$. Figures $5 \mathrm{a}$ and $5 \mathrm{~b}$ show the commanded linear compliance forces on the right and left hand respectively, which reach a maximum of $142 \mathrm{~N}$ on the right and $134 \mathrm{~N}$ on the left hand. Figure $5 \mathrm{c}$ shows the $x$ and $y$ coordinates of the CoM, which is autonomously shifted backwards due to (15) to compensate for the weight of the box. This is also reflected in Fig. 5d where the ZMP, measured from sensors on the feet of the robot, is rapidly stabilized after the disruptions during the application and the release of the weight. The sensors used for measuring the ZMP position are relatively noisy, and this is visible in the initial $(t<3 \mathrm{~s})$ and final $(t>20 \mathrm{~s})$ stages of the experiment. The estimation of the CoM position (calculated from joint encoders as in [5]) is, on the other hand, more smooth and accurate. The trajectories of both ZMP and CoM throughout the experiment are visualized in Fig. 5e over the support polygon. Note that the ZMP remains in the support area throughout the whole experiment. Despite the disruptions during the box handover, the ZMP remains almost stationary while the CoM is shifted to the back in order to compensate 


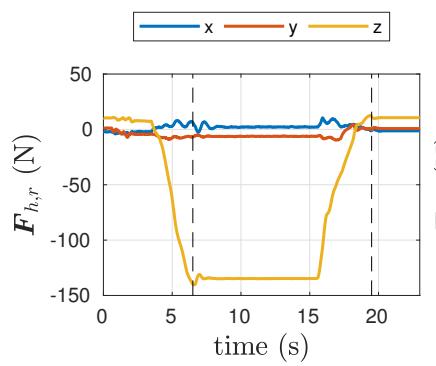

(a)

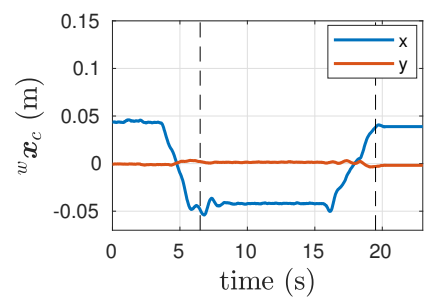

(c)
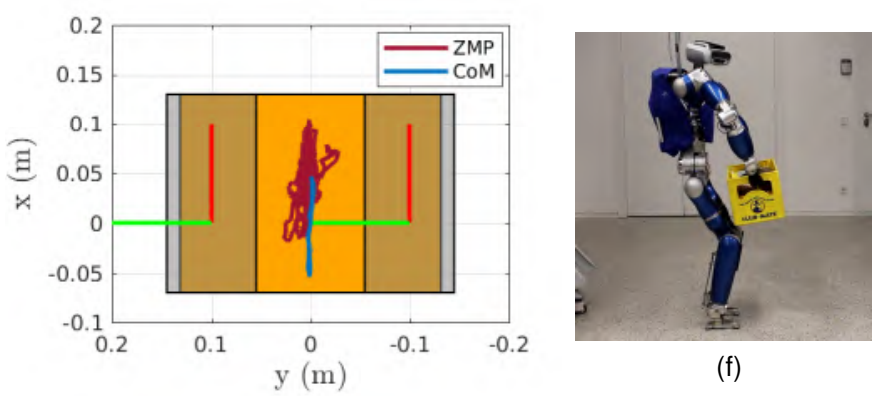

(f)

(e)

Fig. 5. Results for experiment 1: lifting a box of $25 \mathrm{~kg}$. (a) Force on the right hand. (b) Force on the left hand. (c) CoM position. (d) ZMP position. (e) CoM and ZMP over the support polygon. (f) Experimental setup.

for the weight of the box. It is worth noting here that the maximum weight of the box was limited by the maximum torques that the joints can deliver (in particular, the limit of $40 \mathrm{Nm}$ at the elbow was critical). In the attached video, it is visible that the elbows were stretched when carrying the whole mass of $25 \mathrm{~kg}$.

2) Experiment 2. Table Pushing: In the second experiment, the robot is placed in front of a table and is required to push it forward. The weight of the table and the friction parameters with the ground are unknown (as it is usually the case in such situations), the push-forward motion is planned as a forward Cartesian trajectory for the two hands with no feedforward forces. Figures $6 \mathrm{a}$ and $6 \mathrm{~b}$ show the commanded linear compliance forces, which reach an overall magnitude of $79.5 \mathrm{~N}$ and $60.8 \mathrm{~N}$ on the right and left hands, respectively. Figure 6c shows the $x$ and $y$ coordinates of the CoM, which shifts significantly forward to compensate for the forces applied by the interacting end-effectors. In fact, the CoM leaves the ground support area toward the front, which would result in an immediate fall without the interaction forces. This is reflected in Fig. 6d and Fig. 6e, which depict the trajectory of the ZMP. Note that the contact configuration, and therefore the ZMP support area, is different with respect to the first experiment.

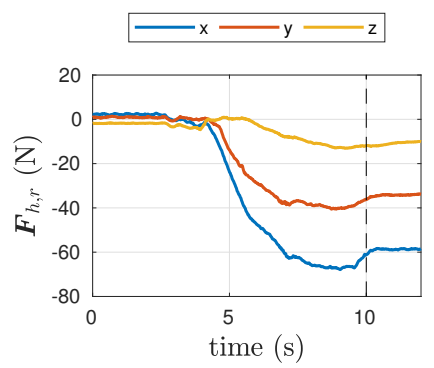

(a)

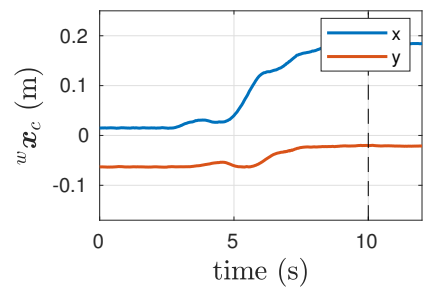

(c)

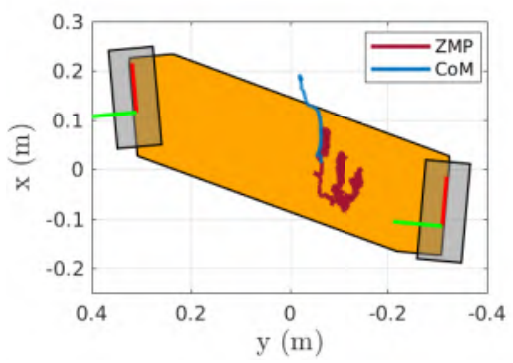

(e)

Fig. 6. Results for experiment 2: pushing a table weighing $50 \mathrm{~kg}$. (a) Force on the right hand. (b) Force on the left hand. (c) CoM position. (d) ZMP position. (e) CoM and ZMP over the support polygon. (f) Experimental setup.

Figure 6e shows that the ZMP is significantly far from the boundaries of its support area throughout the experiment. It is slightly shifted from the center due to noisy measurements and minor uncertainties in the model of the robot.

3) Experiment 3. Interacting with the Right Foot: The robot in this experiment is balancing using its left foot and right hand, while the right foot and the left hand are free endeffectors available for interaction. The robot uses its right foot to push an object (a fire extinguisher of mass $19.8 \mathrm{~kg}$ ). Again, the environment and its properties are unknown to the controller, and the motion of the foot is simply planned in Cartesian space. Figure 7 describes the results of the performed experiments. Figure 7a shows the commanded compliance forces on the right foot, which increase in magnitude up to $70.3 \mathrm{~N}$. The different phases of the experiment are clearly distinguishable on the graph as the foot establishes contact with the object (force starts to increase), starts pushing it (the force is at its maximum) and finally retreats back to its initial position (the force goes back to zero). Figure $7 \mathrm{~b}$ shows the forces applied by the right hand, which is used for balancing. Note that the applied force maintains a stable behavior as the right foot pushes and releases the object. The threshold for the maximum balancing forces to be applied on the right hand is $100 \mathrm{~N}$; this threshold is never reached during the experiment. 


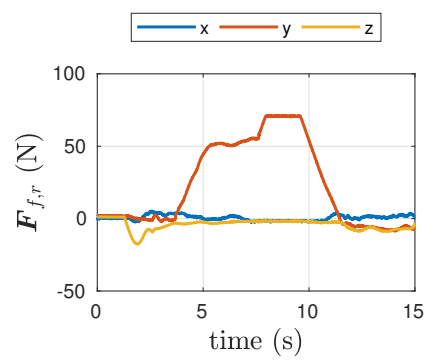

(a)

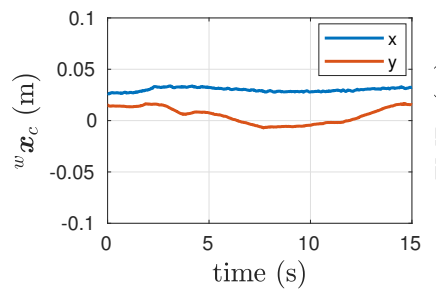

(c)

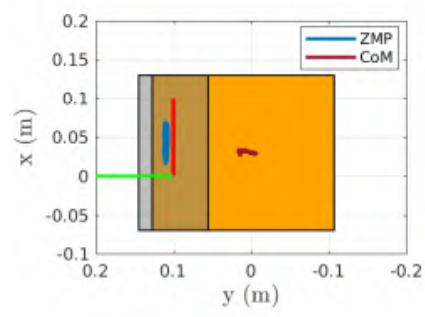

(e)

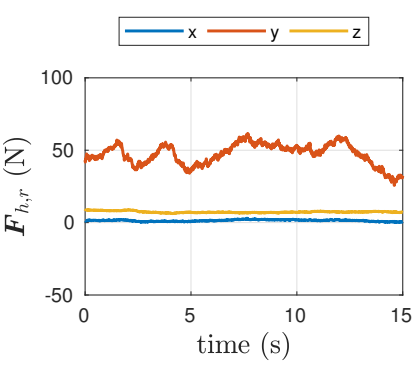

(b)

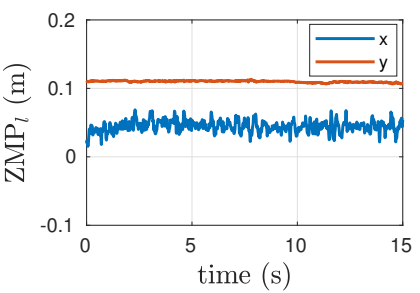

(d)

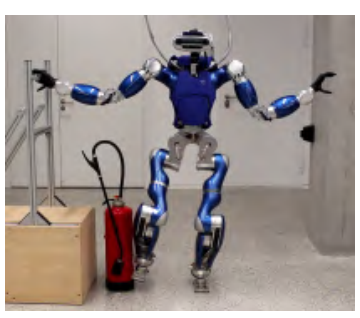

(f)
Fig. 7. Results for experiment 3: using the right foot to move a heavy fire extinguisher $(19.8 \mathrm{~kg})$. (a) Force on the right hand. (b) Force on the left hand (c) CoM position. (d) ZMP position. (e) CoM over the support area (orange) and ZMP of the left foot. (f) Experimental setup.

The motion of the CoM as it counteracts the force applied on the foot by moving slightly to the right is depicted in Fig. 7c and Fig. 7e. The latter shows the trajectory of the CoM over the support area, which is calculated from the contact configuration of the balancing end-effectors (the left foot and the right hand in this case). The left foot, which maintains contact with the ground, is shown in gray. As there is no information about the forces applied on the right hand (no force/pressure sensors available), the ZMP trajectory plotted in Fig. 7e corresponds to the ZMP of the left foot only (measured with the force-torque sensor in the foot). The mentioned ZMP keeps a relatively constant position throughout the experiment, regardless of the forces applied by the right foot.

4) Experiment 4. Automatic Contact Switching: The fourth experiment aims at validating the automatic contact switching algorithm described in section III-C. Before the beginning of the experiment, the robot (standing on its feet) establishes contact with the structure using its right hand. All three endeffectors (the right hand and both feet) remain in contact throughout the experiment (Fig. 8). At $\mathrm{t}=0 \mathrm{~s}$, the robot balances on its two feet with no load on its right hand. It then incorporates its right hand as a balancing end-effector and shifts its weight to balance on its feet and its right hand $(\mathrm{t}=7 \mathrm{~s})$. Finally, it shifts its weight again to balance on its right hand and its left foot, with no load on its right foot

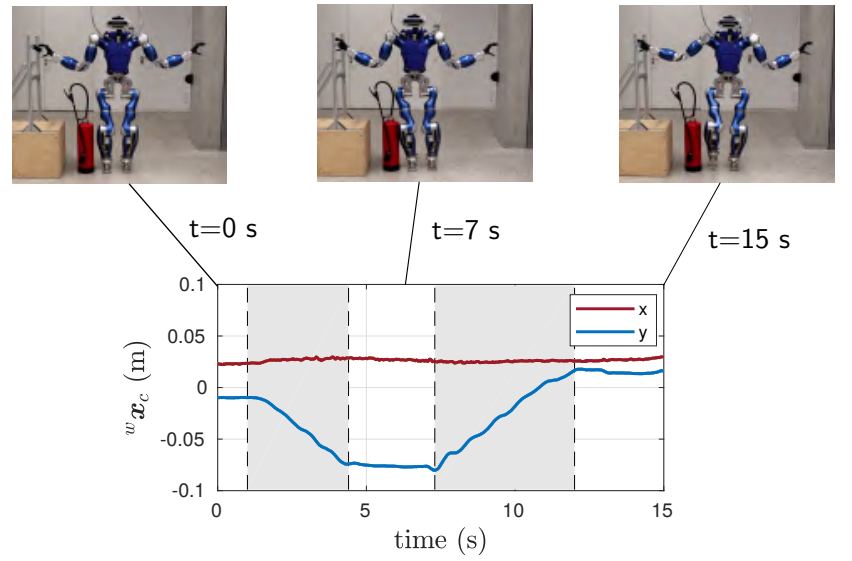

(a)

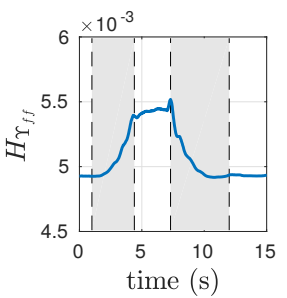

(b)

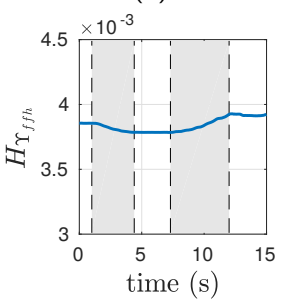

(c)

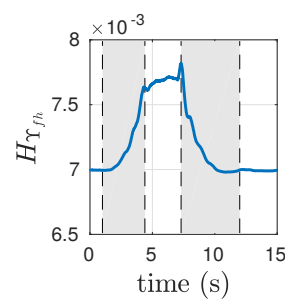

(d)
Fig. 8. Results for experiment 4: The robot shifts its balance autonomously between the three end-effectors that are in contact. It switches between contact configuration $\Upsilon_{f f}$ (only the two feet used for balancing), contact configuration $\Upsilon_{f f h}$ (the two feet and the right hand all used for balancing), and contact configuration $\Upsilon_{f h}$ (only the left foot and the right hand used for balancing). Figure (a) shows the change in position for the CoM. Figures (bd) show the cost function $H$ corresponding to contact configurations $\Upsilon_{f f}$, $\Upsilon_{f f h}$ and $\boldsymbol{\Upsilon}_{f h}$ respectively. A lower value of $H$ reflects a better balance of the robot.

$(\mathrm{t}=15 \mathrm{~s})$. Figure $8 \mathrm{a}$ shows the change in the CoM position as the robot shifts from one balancing configuration to another one. The three contact configurations are respectively referred to by $\boldsymbol{\Upsilon}_{f f}$ for balancing on two feet, $\boldsymbol{\Upsilon}_{f f h}$ for balancing on both feet and the right hand, and $\boldsymbol{\Upsilon}_{f h}$ for balancing on the left foot and the right hand. Since a different GIWC exists for each contact configuration, the value of the cost function $H$, depicting the stability of the robot, is different depending on which GIWC is considered, even if the state of the robot did not change. For example, the robot would be more stable when using all three end-effectors for balancing rather than using only two (with the third being in contact but carrying no weight). This is reflected in Fig. 8b-8d, where the evolution of the potential $H$ for each configuration is shown. The three figures depict $H$ for contact configurations $\Upsilon_{f f}$, $\boldsymbol{\Upsilon}_{f f h}$ and $\boldsymbol{\Upsilon}_{f h}$ respectively. Note that a lower $H$ reflects a better stability. The regions shaded in gray represent the phases during which the balance switch takes place. During the first phase ( $\mathrm{t}=1 \rightarrow 4.4 \mathrm{~s}$ ) the robot, which was balancing on its two feet with no load on its right hand, incorporates that hand as an additional balancing end-effector. During the second phase $(\mathrm{t}=7.3 \rightarrow 12 \mathrm{~s})$ the robot shifts from balancing on its two feet and its right hand to balancing on its left foot and its right hand, removing all load from the right foot (which can now be lifted if needed).

The results of the experiment showed several interesting characteristics of the architecture and of the proposed potential 
function $H$. First, note that the average value of $H$ is lowest for contact configuration $\Upsilon_{f f h}$ at $\approx 3.8 \mathrm{e}-3$ (Fig. 8b). It is higher for contact configuration $\Upsilon_{f f}$, where it ranges between 4.8e-3 and 5.5e-3 (Fig. 8a), and it is highest for contact configuration $\Upsilon_{f h}$, where $H$ reaches a maximum of $7.8 \mathrm{e}-3$ (Fig. 8c). Recalling from the definition of $H$ in eq. (13) that the lower the potential the better the balance, the results go inline with the intuition that the robot is best balanced using all three end-effectors, and least balanced when balancing on one foot and a hand only. On the other hand, the contact switch is reflected as the robot shifts from configuration $\boldsymbol{\Upsilon}_{f f}$ to $\boldsymbol{\Upsilon}_{f f h}$, where the potential corresponding to $\Upsilon_{f f}$ increases while that of $\Upsilon_{f f h}$ decreases to its minimum. A similar behavior is observed when switching from $\boldsymbol{\Upsilon}_{f f h}$ to $\boldsymbol{\Upsilon}_{f h}$, where the potential corresponding to the former increases while that of the latter decreases to its minimum. On the other hand, the degree of variation of each potential reflects as well the robustness of each balancing configuration. We notice that balancing on one foot and one hand is quite sensitive to changes in the posture of the robot, whereas balancing on both feet and the hand is altogether a much more robust posture.

\section{Conclusions}

In this paper, we have presented a controller for maintaining the balance of torque-controlled humanoid robots in the presence of unknown (and high) external forces. The controller acts at the wrench level to ensure that the needed balancing forces lie within the Gravito-Inertial Wrench Cone (GIWC). The same approach is applied to allow for automatic switching between different contact configurations by acting on the GIWC itself. The efficiency and robustness of the approach was demonstrated by several experiments that tested the robot hardware with forces up to the order of $250 \mathrm{~N}(\approx 1 / 3$ of the robot's weight). The robot interacted with and manipulated the environment using one hand, both hands and even one foot, while the controller was handling the different force directions and contact configurations.

An extension of the present work is the consideration of the tracking case in order to ensure balance of the robot in highly dynamic scenarios. Actuator limits could be included into the GIWC following the ideas in [21]. Moreover, it is interesting to couple the controller with a higher-level planner to allow for complicated tasks like opening spring-loaded doors. A more exhaustive analysis of passivity and stability of the proposed architecture is a future work.

\section{REFERENCES}

[1] A. Ibanez, P. Bidaud, and V. Padois, "Unified preview control for humanoid postural stability and upper-limb interaction adaptation," in IEEE/RSJ Int. Conf. Intelligent Robots and Systems, 2012, pp. 18011808.
[2] C. Ott, B. Henze, and D. Lee, "Kinesthetic teaching of humanoid motion based on whole-body compliance control with interaction-aware balancing," in IEEE/RSJ Int. Conf. Intelligent Robots and Systems, 2013, pp. $4615-4621$.

[3] L. Sentis, "Compliant control of whole-body multi-contact behaviors in humanoid robots," in Motion Planning for Humanoid Robots, K. Harada, E. Yoshida, and K. Yokoi, Eds. Springer, 2010, pp. 29-66.

[4] B. Henze, C. Ott, and M. A. Roa, "Posture and balance control for humanoid robots in multi-contact scenarios based on model predictive control," in IEEE/RSJ Int. Conf. Intelligent Robots and Systems, 2014, pp. 3253-3258.

[5] B. Henze, M. A. Roa, and C. Ott, "Passivity-based whole-body balancing for torque-controlled humanoid robots in multi-contact scenarios," Int. J. Robotics Research, vol. 35, no. 12, pp. 1522 - 1543, 2016.

[6] B. Henze, A. Dietrich, and C. Ott, "An approach to combine balancing with hierarchical whole-body control for legged humanoid robots," IEEE Robotics and Automation Letters, vol. 1, no. 2, pp. 700 - 707, 2016.

[7] B. Henze, A. Dietrich, M. A. Roa, and C. Ott, "Multi-contact balancing of humanoid robots in confined spaces: Utilizing knee contacts," in IEEE/RSJ Int. Conf. Intelligent Robots and Systems, 2017, pp. 697-704.

[8] B. J. Stephens and C. G. Atkeson, "Push recovery by stepping for humanoid robots with force controlled joints," in IEEE-RAS Int. Conf. Humanoid Robots, 2010, pp. $52-59$.

[9] L. Righetti, J. Buchli, M. Mistry, M. Kalakrishnan, and S. Schaal, "Optimal distribution of contact forces with inverse dynamics control," Int. J. Robotics Research, vol. 32, no. 3, pp. 280 - 298, 2013.

[10] M. Mistry, J. Buchli, and S. Schaal, "Inverse dynamics control of floating base systems using orthogonal decomposition," in IEEE Int. Conf. Robotics and Automation, 2010, pp. $3406-3412$.

[11] S. Hyon, J. Hale, and G. Cheng, "Full-body compliant human-humanoid interaction: Balancing in the presence of unknown external forces," IEEE Trans. Robotics, vol. 23, no. 5, pp. 884-898, 2007.

[12] C. Ott, M. A. Roa, and G. Hirzinger, "Posture and balance control for biped robots based on contact force optimization," in IEEE-RAS Int. Conf. Humanoid Robots, 2011, pp. 26-33.

[13] A. Escande, N. Mansard, and P.-B. Wieber, "Hierarchical quadratic programming: Fast online humanoid-robot motion generation," Int. J. Robotics Research, vol. 33, pp. 1006-1028, 2014.

[14] M. Murooka, S. Nozawa, Y. Kakiuchi, K. Okada, and M. Inaba, "Wholebody pushing manipulation with contact posture planning of large and heavy object for humanoid robot," in IEEE Int. Conf. Robotics and Automation, 2015, pp. 5682-5689.

[15] S. Caron and A. Kheddar, "Multi-contact walking pattern generation based on model preview control of 3D COM accelerations," in IEEERAS Int. Conf. Humanoid Robots, 2016, pp. 550 - 557.

[16] S. Caron, Q.-C. Pham, and Y. Nakamura, "Leveraging cone double description for multi-contact stability of humanoids with applications to statics and dynamics," in Robotics: Science and System, 2015.

[17] S. Caron, Q. Pham, and Y. Nakamura, "ZMP support areas for multicontact mobility under frictional constraints," IEEE Trans. on Robotics, vol. 33, no. 1, pp. 67-80, 2017.

[18] A. D. Prete, S. Tonneau, and N. Mansard, "Fast algorithms to test robust static equilibrium for legged robots," in IEEE Int. Conf. on Robotics and Automation, 2016, pp. 1601-1607.

[19] H. Hirukawa, S. Hattori, K. Harada, S. Kajita, K. Kaneko, F. Kanehiro, K. Fujiwara, and M. Morisawa, "A universal stability criterion of the foot contact of legged robots - adios ZMP," in IEEE Int. Conf. Robotics and Automation, 2006, pp. 1976-1983.

[20] J. Englsberger, A. Werner, C. Ott, B. Henze, M. A. Roa, G. Garofalo, R. Burger, A. Beyer, O. Eiberger, K. Schmid, and A. Albu-Schäffer, "Overview of the torque-controlled humanoid robot TORO," in IEEERAS Int. Conf. Humanoid Robots, 2014, pp. 916-923.

[21] R. Orsolino, M. Focchi, C. Mastalli, H. Dai, D. G. Caldwell, and C. Semini, "Application of wrench based feasibility analysis to the online trajectory optimization of legged robots," IEEE Robotics and Automation Letters, vol. 3, no. 4, pp. 3363 - 3370, 2018. 